\title{
Haloxylon ammodendron (Amaranthaceae) fruit development delay caused by post-flowering non-inductive photoperiod
}

\author{
REN Cai ${ }^{1}$, YU Tian², QU Guanghang ${ }^{1}$, WANG Shuang ${ }^{1}$, WANG Ze ${ }^{3}$, Abudoukeyumu \\ MIJITI $^{3}$, ZHANG Hua $^{3}, \mathrm{MA} \mathrm{Lin}^{3}$, HE Xiaoling ${ }^{1}$, MA Hao ${ }^{1,3^{*}}$ \\ ${ }^{1}$ State Key Laboratory of Crop Genetics and Germplasm Enhancement, Nanjing Agricultural University, Nanjing 210095, \\ China; \\ ${ }^{2}$ Urumqi County Agricultural Technology Extension Center, Urumqi 830011, China; \\ ${ }^{3}$ Desert Research Institute in the Arid Region, Xinjiang Agricultural University, Urumqi 830052, China
}

\begin{abstract}
Haloxylon ammodendron (C. A. Mey.) is one of the economically and ecologically important desert trees used for sand fixation. The ovary of $H$. ammodendron is found not to swell after flowering in spring until at the end of August or early September in western China. However, what happens for ovary at anatomic level in that period and which crucial ecological factor regulates the phenomenon of $H$. ammodendron have not been fully understood. To characterize the phenomenon and explore the crucial environmental regulating factors, we carried out the morphological and anatomic observations at the different development stages of the fruits and three single-factor experiments (low air temperature, sufficient soil moisture, and short day length). Our results showed that under the natural conditions, the ovary of $H$. ammodendron after flowering developed slowly and the morphological changes of fruits were not significant for the period from May to August and after late August or early September; and then the ovary developed rapidly and matured in October. Cell division in embryo was observed to start approximately 25 days after flowering (DAF) and just developed to globular embryo stage at mid-August. Photoperiod was identified as the pivotal environmental factor regulating the fruit development of H. ammodendron. Moreover, the threshold value of day length for the fruit development was $14.0 \mathrm{~h}$. A long day $(>14.0 \mathrm{~h})$ treatment began from $5 \mathrm{DAF}$ could delay fruit development of $H$. ammodendron while a short day $(<14.0 \mathrm{~h})$ treatment could accelerate it. Moreover, a further longer day treatment $(>15.0 \mathrm{~h})$ could also delay fruit development even when they had developed for a long time (110 DAF). The present study indicated that $H$. ammodendron adopted a reproductive strategy of delayed fruit development and this strategy helps it survive and obtain offspring in harsh desert habitats.
\end{abstract}

Keywords: environmental factor; fruit development; Haloxylon ammodendron; photoperiod; reproductive strategy

Citation: REN Cai, YU Tian, QU Guanghang, WANG Shuang, WANG Ze, Abudoukeyumu MIJITI, ZHANG Hua, MA Lin, HE Xiaoling, MA Hao. 2017. Haloxylon ammodendron (Amaranthaceae) fruit development delay caused by post-flowering non-inductive photoperiod. Journal of Arid Land, 9(3): 408-418. doi: 10.1007/s40333-017-0093-4

\section{Introduction}

Haloxylon ammodendron belongs to Haloxylon Bunge (Amaranthaceae) that consists of 11 species identified so far worldwide. The geographical distribution of $H$. ammodendron is concentrated in temperate and subtropical desert areas of Africa and Asia (Pyankov et al., 1999; Tobe et al., 2000; Sheng et al., 2005). In China, the total distributing acreage of $H$. ammodendron

\footnotetext{
*Corresponding author: MA Hao (E-mail: Lqncsi@njau.edu.cn)

The first and second authors contributed equally to this work.

Received 2016-07-24; revised 2017-01-25; accepted 2017-04-04

(C) Xinjiang Institute of Ecology and Geography, Chinese Academy of Sciences, Science Press and Springer-Verlag Berlin Heidelberg 2017
} 
is approximately $8.22 \times 10^{6} \mathrm{hm}^{2}$. Its largest distribution acreage is in Xinjiang, accounting for $62.7 \%$ of China's total distribution acreage, followed by $19.6 \%$ in Inner Mongolia, $10.9 \%$ in Qinghai, and 6.8\% in Gansu (Guo et al., 2009). H. ammodendron is one of economically important and ecologically vulnerable desert tree species used for sand fixation in China (Wang et al., 2009; Yang et al., 2014). It plays an important role in maintenance of the structure and function of desert ecosystems, reducing wind speed and ameliorating the microclimate, thus facilitating the settlement and growth of other desert plants (Shamsutdinov and Ubaidullaev, 1988).

The reproduction of plant is important in its life cycle and is affected by genetic and/or environmental factors (Brock, 1983). Environmental characteristics of desert is always complicated and varied. The Gurbantunggut Desert, the largest stable and semi-stable sand desert in China with $H$. ammodendron as the major dominant species of the community composition, has a continental climate; the mean annual potential evaporation exceeds $2200 \mathrm{~mm}$ while the mean annual precipitation is only $70-150 \mathrm{~mm}$ falling predominantly during spring (March to May). The annual mean temperature is $6^{\circ} \mathrm{C}-10^{\circ} \mathrm{C}$. It is torrid in summer (June to August) with the maximum monthly mean temperature of $32.6^{\circ} \mathrm{C}$ in July and the light intensities more than $1800 \mu \mathrm{mol} /\left(\mathrm{m}^{2} \cdot \mathrm{s}\right)$ in clear days; and is cold in winter with the minimum monthly mean temperature of $-21.8^{\circ} \mathrm{C}$ in January. The annual daylight hours ranged from $8.8 \mathrm{~h}$ on 21 December to $15.5 \mathrm{~h}$ on 21 June (Zhang et al., 2007; Li et al., 2010). To adapt to the torrid summer, many plants in the desert have developed different reproductive strategies. For example, an annual ephemeral plant Diptychocarpus strictus (Brassicaceae) flowers from mid-April to early May, and seeds mature in late May to early June to cope with the harsh and fickle environment to achieve reproductive success before the torrid summer comes ( $\mathrm{Lu}$ et al., 2010). Another example is Tamarix ramosissima (Tamaririxaceae). This perennial plant has two distinct flowering periods (one in spring and another in summer) to adapt to the instable climate conditions (Yan et al., 2011). Nevertheless, $H$. ammodendron is found to be of a different reproductive strategy. The flowering period of $H$. ammodendron occurs from late April to mid-May with a duration less than half a month. However, after blooming, the ovary of $H$. ammodendron almost does not swell until late August or early September. Afterwards, its ovary begins to enlarge rapidly and fruit wings are visible, and the fruit matures in October (Tursunov et al., 1989; Zhang, 2002; Sheng et al., 2003; Wei and Wang, 2006).

Zhang (2002) suggested that ovary of $H$. ammodendron almost does not swell after flowering for a long time and reasons for non-swelling might be related with high air temperature and/or low moisture content in soil in summer. However, under the appropriate greenhouse growth conditions $\left(10^{\circ} \mathrm{C}-30^{\circ} \mathrm{C}\right.$, sufficient light and water), well-grown plants of $H$. ammodendron were also found to display the same phenomenon (Yu, 2012). What is the actual environmental factor that induces $H$. ammodendron to adopt this reproductive strategy? In the present study, we conducted morphological and anatomic observations on the fruits and designed three single-factor experiments (low air temperature, sufficient moisture supply in soil, and short day) to characterize the phenomenon and explore the crucial environmental regulation factor.

\section{Materials and methods}

\subsection{Study area}

The study area is in Xinjiang Uygur Autonomous Region, China. We selected three field observation sites (A, B and C in Fig. 1) to conduct the research. Site A $\left(44.4^{\circ} \mathrm{N}, 87.9^{\circ} \mathrm{E}\right)$ is a native desert habitat of $H$. ammodendron; site B $\left(43.5^{\circ} \mathrm{N}, 87.0^{\circ} \mathrm{E}\right)$ is in Shuixigou Village, where a greenhouse can provide good experimental conditions; and site $\mathrm{C}\left(44.2^{\circ} \mathrm{N}, 87.3^{\circ} \mathrm{E}\right)$ is in Wutonggou Village, where the illumination treatment experiments were conducted. Site A and C were close to the south margin of the Gurbantunggut Desert.

\subsection{Fruit characteristic}

The fruit development of $H$. ammodendron under natural growth conditions was investigated at 
Site A. Fruits were collected randomly from 10 plants in the field from flowering stage to maturation stage. The anatomic dissection was carried out using the paraffin method: fixed in formalin/acetic acid/alcohol (70\% alcohol/glacial acetic acid/formalin, 18/1/1 by volume) fixation. The blocks were sectioned at $8-12 \mu \mathrm{m}$ and routinely stained with safranin and fast green (Ma et al., 2004; Li, 2009). The sectioned samples were observed under a light microscope of Leica DM 2500 (Leica, Wetzlar, Germany). The differences of fruit size at the different development stages were analyzed for normality and homogeneity of variance prior to fulfill the requirements of T-tests and one-way analysis of variance (ANOVA). The variances were found not to be homogeneous, and then were transformed using log10 or square root. The transformed data, however, were still found not to be homogenous. Therefore, the multiple comparisons of the fruit sizes of the different development stages were assessed using a more conservative Kruskal-Wallis non-parametric test with a significance level of $P<0.05$. Data analysis was performed with the software IBM SPSS Statistics 19.0.

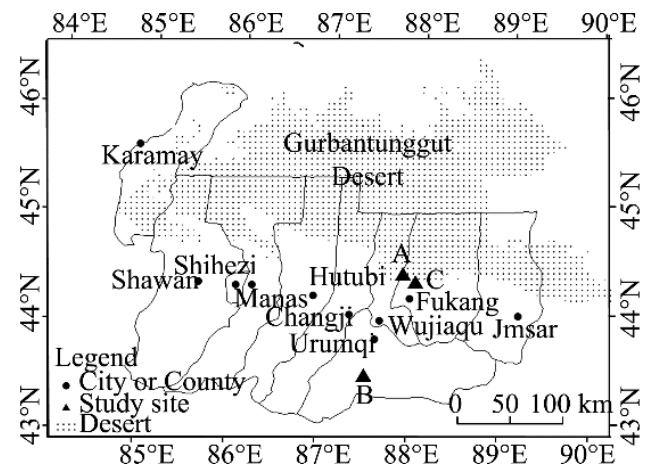

Fig. 1 Locations of the field observation sites: A $\left(44.4^{\circ} \mathrm{N}, 87.9^{\circ} \mathrm{E}\right), \mathrm{B}\left(43.5^{\circ} \mathrm{N}, 87.0^{\circ} \mathrm{E}\right)$ in Shuixigou Village and $\mathrm{C}\left(44.2^{\circ} \mathrm{N}, 87.3^{\circ} \mathrm{E}\right)$ in Wutonggou Village

\subsection{Plant materials and planting}

Two-year old plants of $H$. ammodendron were transplanted in spring to plastic nutrition pots (6 L; one plant per pot) filled with culture medium (desert sand/vermiculite/perlite/decaying sheep excrement, 5.0/1.5/1.5/2.0 by volume). All the potted plants were watered as the normal with the soil moisture content maintained at approximately 9\% (Yu et al., 2012). After flowering, all the plants were used for further studies.

\subsection{Single-factor experiments}

To reveal the underlying environmental factors causing the slow post-flowering reproductive development (SPRD) of the $H$. ammodendron ovary, we carried out three single-factor (low temperature, sufficient moisture supply in soil, and short day) experiments within greenhouses at Shuixigou Village. We divided 120 two-year-old plants (after flowering) into four groups (Group-A) with 30 plants per group (Table 1). Group-A I was taken as the control. The plants were growing in a temperature-controlled greenhouse (day, $30 \pm 2^{\circ} \mathrm{C}$; night, $10 \pm 2^{\circ} \mathrm{C}$; natural light intensity, $\left.900-1260 \mu \mathrm{mol} /\left(\mathrm{m}^{2} \cdot \mathrm{s}\right)\right)$, with other conditions same as those set in Section 2.3 . The photon flux density was measured at the canopy level with LI-6400 portable photosynthetic system (LI-COR, Lincoln, NE, USA). Group-A II was treated with low air temperature in a plastic shack with an air-conditioner, and other measures were same as the group I. Group-A III was supplied with sufficient moisture in soil and the soil moisture content was maintained at approximately 20\%, with other measures same as the Group-A I too. The sunshine duration for Group-A IV was shortened to $10.0 \mathrm{~h} / \mathrm{d}$, with other measures same as the group I as well. For all the groups, day length of $10.0 \mathrm{~h} / \mathrm{d}$ treatment was conducted by transferring the pots into and out of a dark room every day (19:00 to 09:00 next day inside; light intensity $<1 \mu \mathrm{mol} /\left(\mathrm{m}^{2} \cdot \mathrm{s}\right)$; Cheng et al., 2011), and temperature difference was within a range of $1^{\circ} \mathrm{C}$. All treatments were conducted from 5 to 85 DAF. 
Table 1 Environmental factor treatments

\begin{tabular}{cclc}
\hline Group-A & Number of plants & \multicolumn{1}{c}{ Treatment } & Treatment stage (DAF) \\
\hline I & 30 & ND (approximately 14.0 h during flowering, 15.5 h on & $5-85$ \\
II & 30 & 21 June and 14.0 h on 14 August) & $5-85$ \\
III & 30 & Low air temperature (day, 20 $\pm 2^{\circ} \mathrm{C}$; night, $\left.5 \pm 2^{\circ} \mathrm{C}\right)$ & $5-85$ \\
IV & 30 & Sufficient moisture supply in soil (approximately 20\%) & $5-85$ \\
\hline
\end{tabular}

Note: ND, natural day length; SD, short day; DAF, days after flowering; I, control; II, temperature; III, moisture; IV, daylight.

\subsection{Day length threshold of the fruit development}

The illumination treatment experiments were conducted at Site C in Wutonggou Village. We divided 120 plants (after flowering) into four groups (Group-B) with 30 plants per group (Table 2). Group-B I, II and III were subjected to different illumination treatments of 13.5, 14.0 and 14.5 $\mathrm{h} / \mathrm{d}$, respectively, by an artificial lighting instrument automatically turned on and off at the pre-set time. The light sources were provided by incandescent bulbs, which provided a light intensity about $65 \mu \mathrm{mol} /\left(\mathrm{m}^{2} \cdot \mathrm{s}\right)$ at the canopy of the plants that is close to and lower than the light compensation point of $H$. ammodendron (Su et al., 2007; Xu et al., 2007). The illumination was selected on the base of the day length of $13.5 \mathrm{~h}$ on 25 August at the south of Gurbantunggut Desert $\left(44.4^{\circ} \mathrm{N}, 87.9^{\circ} \mathrm{E}\right)$ when the ovary of $H$. ammodendron began to swell in the local field. Group-B IV was used as control under natural sunshine duration. All treatments were conducted from 5 to 85 DAF.

Table 2 Determination of threshold value of day length for the development of fruits in H. ammodendron

\begin{tabular}{cccc}
\hline Group-B & Number of plants & Illumination (h/d) & Treatment stage (DAF) \\
\hline I & 30 & 13.5 & $5-85$ \\
II & 30 & 14.0 & $5-85$ \\
III & 30 & 14.5 & $5-85$ \\
IV & 30 & ND & $5-85$ \\
\hline
\end{tabular}

Note: I, II and III, illumination treatments of 3.5, 14.0 and 14.5 h/d, respectively; IV, the control.

\subsection{Effects of different illumination treatments}

To further explore the effects of different illumination on the fruit development of $H$. ammodendron, we conducted three different day length treatments (Table 3). Group-C I was treated from 5 to 110 DAF with a day length of $10.0 \mathrm{~h} / \mathrm{d}$. Group-C II was exposed to natural day length (ND) as control from 0 to 170 DAF. For Group-C III, all the plants after flowering were remained under ND until $109 \mathrm{DAF}$, and then, was placed under a $15.0 \mathrm{~h} / \mathrm{d}$ long day (LD) treatment (from 07:00 to 22:00) from 110 to 170 DAF.

Table 3 Different illumination treatments

\begin{tabular}{cccc}
\hline Group-C & Number of plant & Illumination & Treatment stage (DAF) \\
\hline I & 30 & SD (10.0 h/d) & $5-110$ \\
II & 30 & ND & $0-170$ \\
III & 30 & LD (15.0 h/d) & $110-170$ \\
\hline
\end{tabular}

Note: ND, natural day length; SD, short day; LD, long day.

\section{Results}

\subsection{Morphological and anatomic characteristics of fruits}

To reveal the reproductive development progress after flowering under natural growth conditions, morphological and anatomic observations of fruits were carried out. The results showed that after flowering, the ovary of $H$. ammodendron developed slowly from late May to mid-August (Figs. 2a-d and 3). 
Embryonic cell division was observed approximately on 25 DAF (Fig. 2b) and developed to the globular embryo stage on 110 DAF (Fig. 2d). Though the embryo had almost doubled in size (Fig. 2d) compared to that of 25 DAF, the ovary did not display any significant changes $(P<0.05)$ in size from flowering (0 DAF) to the early stage of ovary enlargement (110 DAF; Fig. 4). When
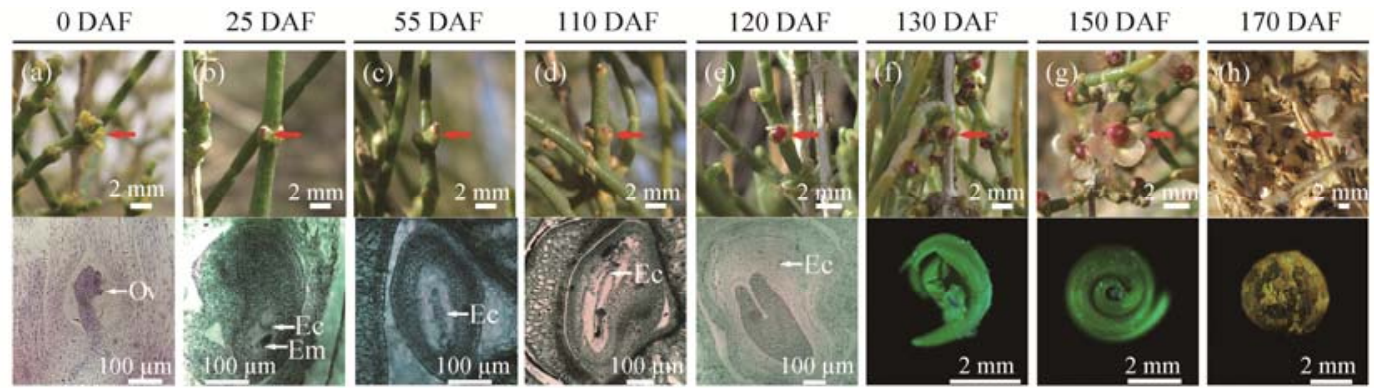

Fig. 2 Morphological and anatomic characteristics of $H$. ammodendron fruit development at site A $\left(44.4^{\circ} \mathrm{N}\right.$, $87.9^{\circ} \mathrm{E}$ ) under natural conditions. Ov, ovule; Em, embryo; Ec, endosperm cell; Red arrows indicate fruits. (a) 0 DAF, blooming period; (b-c) the early stage of ovary development; (d) the early stage of ovary enlargement; (e) the ovary enlargement stage; (f) the early stage of fruit wing development; (g) the fruit wing development stage; (h) the fruit maturation stage (mature seed and fruit).

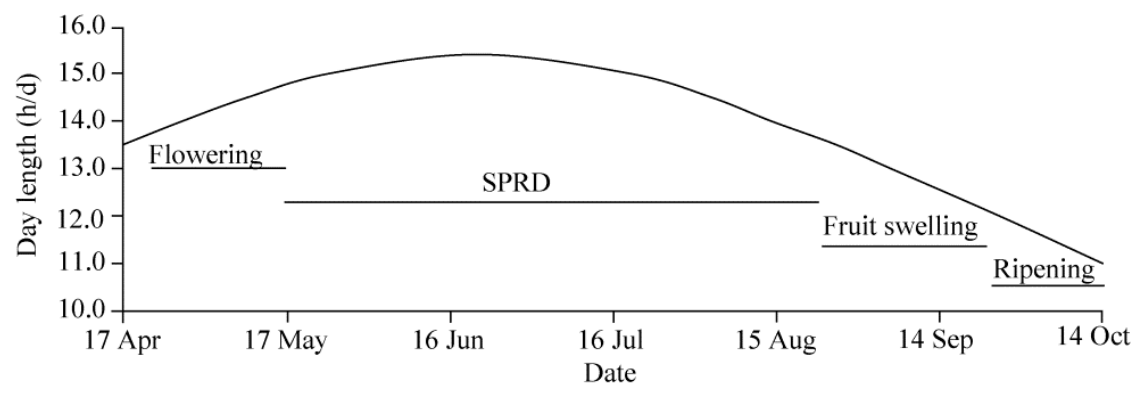

Fig. 3 Day length and scheme of the chronological progression of principal fruit developing stages of $H$. ammodendron at site $\mathrm{A}\left(44.4^{\circ} \mathrm{N}, 87.9^{\circ} \mathrm{E}\right)$. SPRD, slow post-flowering reproductive development.

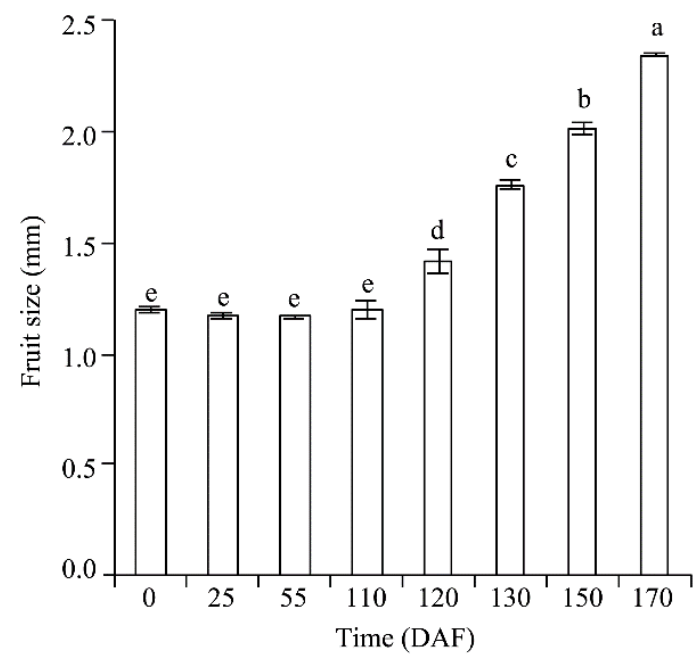

Fig. 4 Fruit size variation of $H$. ammodendron grown under natural day length. The bars represent standard errors of the means, $n=10$. The lowercase letters indicate significant differences at $P<0.05$ level according to the non-parametric test by Kruskal-Wallis. 
the day length became shorter than $14.0 \mathrm{~h} / \mathrm{d}$ for several days in late August, the enlargement of ovary was observable (Fig. 2e) and the change in size was significant $(P<0.05 ; 120$ DAF; Fig. 4). At the same time, the embryo developed to be horseshoe-shaped (the ovary enlargement stage). Afterwards, the ovary and embryo developed rapidly throughout the early stage of fruit wing development (130 DAF; Fig. 2f) and the stage of fruit wing development (150 DAF; Fig. 2g); and the fruit then matured on 170 DAF (Fig. 2h). Additionally, the endosperm was observed to have developed into a free nucleus stage (Figs. 2b-d) and into an endosperm cell stage on 120 DAF (Fig. 2e); and then into a disappearance stage with the development of embryo.

Our results indicated that under natural growth conditions, the ovary of $H$. ammodendron would present a slow reproductive development progress after flowering for approximately 4 months (0-120 DAF; Fig. 3). Accordingly, we defined the phenomenon as a slow post-flowering reproductive development (SPRD).

\subsection{Environmental factor causing SPRD phenomenon of $\boldsymbol{H}$. ammodendron}

Normally, environmental factors include temperature, photoperiod, water, air, soil, etc. Temperature, water, and photoperiod are considered as the dominant environmental factors for plant growth in arid region of northwestern China (Zhang, 2008). When $H$. ammodendron presents a SPRD phenomenon from late May to mid-August, the climate is often characterized by high air temperature, low precipitation, and long day. So we postulated that if high air temperature, low moisture in soil, and long day were the environmental factors causing the SPRD phenomenon of $H$. ammodendron, low air temperature, sufficient moisture supply in soil and short day would be unfavorable for the SPRD phenomenon. To validate this hypothesis, in this study, we carried out three single-factor (low air temperature, sufficient moisture supply in soil, and short day) experiments. The results showed that no accelerated reproductive development progress occurred at the end of experiments in Group-A II and III, being the same as in the control. The results indicated that air temperature and soil moisture may not be associated with the observed $H$. ammodendron SPRD phenomenon. Nevertheless, in Group-A IV, when $H$. Ammodendron was exposed to an illumination of $10.0 \mathrm{~h} / \mathrm{d}$, all the plants were found to be accelerated in the fruit development progress after flowering (Table 4). As the treatment time prolonged (40-50 d), the fruits of the treated plants developed gradually from the ovary enlargement stage to the early stage of fruit wing development. When the illumination treatment was sustained for $80 \mathrm{~d}$, all the treated plants were found to be accelerated into the stage of fruit wing development. The results suggested that it is the day length that caused the SPRD phenomenon of $H$. ammodendron.

Table 4 Number of $H$. ammondendron plants with accelerated development progress of fruits when exposed to an illumination of $10.0 \mathrm{~h} / \mathrm{d}$

\begin{tabular}{|c|c|c|c|c|c|}
\hline \multirow[b]{2}{*}{ DAF } & \multirow[b]{2}{*}{ DAIT } & \multicolumn{3}{|c|}{ Plant number } & \multirow{2}{*}{$\begin{array}{l}\text { Percentage } \\
\text { (\%) }\end{array}$} \\
\hline & & $\begin{array}{l}\text { Ovary enlargement } \\
\text { stage }\end{array}$ & $\begin{array}{c}\text { Early stage of fruit wing } \\
\text { development }\end{array}$ & $\begin{array}{l}\text { Fruit wing development } \\
\text { stage }\end{array}$ & \\
\hline 5 & 1 & 0 & 0 & 0 & 0.0 \\
\hline 45 & 40 & 4 & 0 & 0 & 13.3 \\
\hline 55 & 50 & 9 & 17 & 0 & 86.7 \\
\hline 75 & 70 & 0 & 8 & 22 & 100.0 \\
\hline 85 & 80 & 0 & 0 & 30 & 100.0 \\
\hline
\end{tabular}

Note: DAF, day after flowering; DAIT, days after the illumination treatments.

\subsection{Threshold value of day length for the fruit development of $\mathrm{H}$. ammodendron}

Since the photoperiod causes the SPRD phenomenon of H. ammodendron, it is necessary to determine how the day length threshold affects the delayed fruit development (from the delayed development rate to the accelerated rate, or vice versa). Three illumination treatments of 13.5, 14.0 and $14.5 \mathrm{~h} / \mathrm{d}$ were carried out to determine the day length threshold. As shown in Figure 5, a few of $H$. ammodendron plants treated with $13.5 \mathrm{~h} / \mathrm{d}$ were found to be accelerated in reproductive development progress on 55 DAF while $90 \%$ of plants were found to be accelerated on 80 DAF. 
For the $14.0 \mathrm{~h} / \mathrm{d}$ treatment, no plant was found to be accelerated on $55 \mathrm{DAF}$, while $80 \%$ of plants were found to be accelerated on 80 DAF. For the 14.5 h/d treatment, only 3.3\% of plants were found to be accelerated into the ovary enlargement stage on 80 DAF. It can be estimated that 14.0 $\mathrm{h}$ was the most likely day length threshold that can accelerate the reproductive development of $H$. ammodendron.

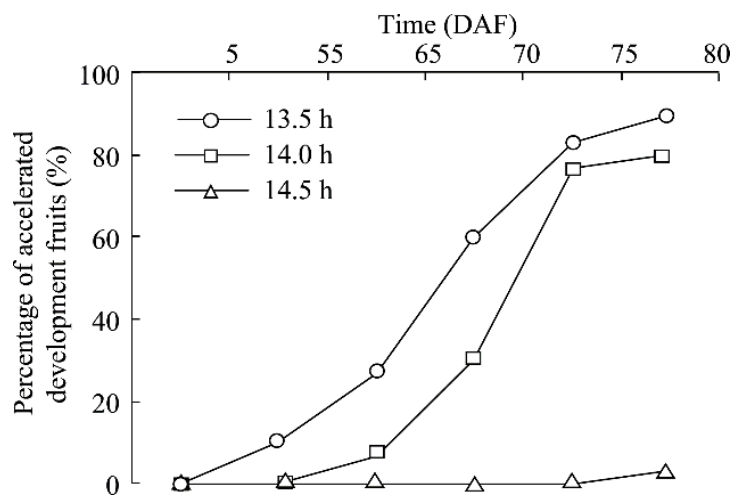

Fig. 5 Percentage of H. ammondendron plants with accelerated development progress of fruits when exposed to different illumination treatments

\subsection{Effects of illumination treatments}

To explore the day length effects on the fruit development of $H$. ammodendron, three day length (SD, ND and LD) treatments were conducted (Table 3). Although there was no obvious difference in fruit size on 25 DAF between the SD and ND treatments, the embryo under SD treatment developed to the pre-embryo stage and its size was twice bigger than that under ND treatment (Fig. 6). Fruit wings began to be visible and embryo gradually enlarged on 55 DAF under SD treatment, while fruits under ND treatment were still at the early stage of ovary development with embryo developing to the pre-embryo stage. Fruits under SD treatment matured on 110 DAF, while embryo under ND treatment had just developed to the globular embryo stage. These results

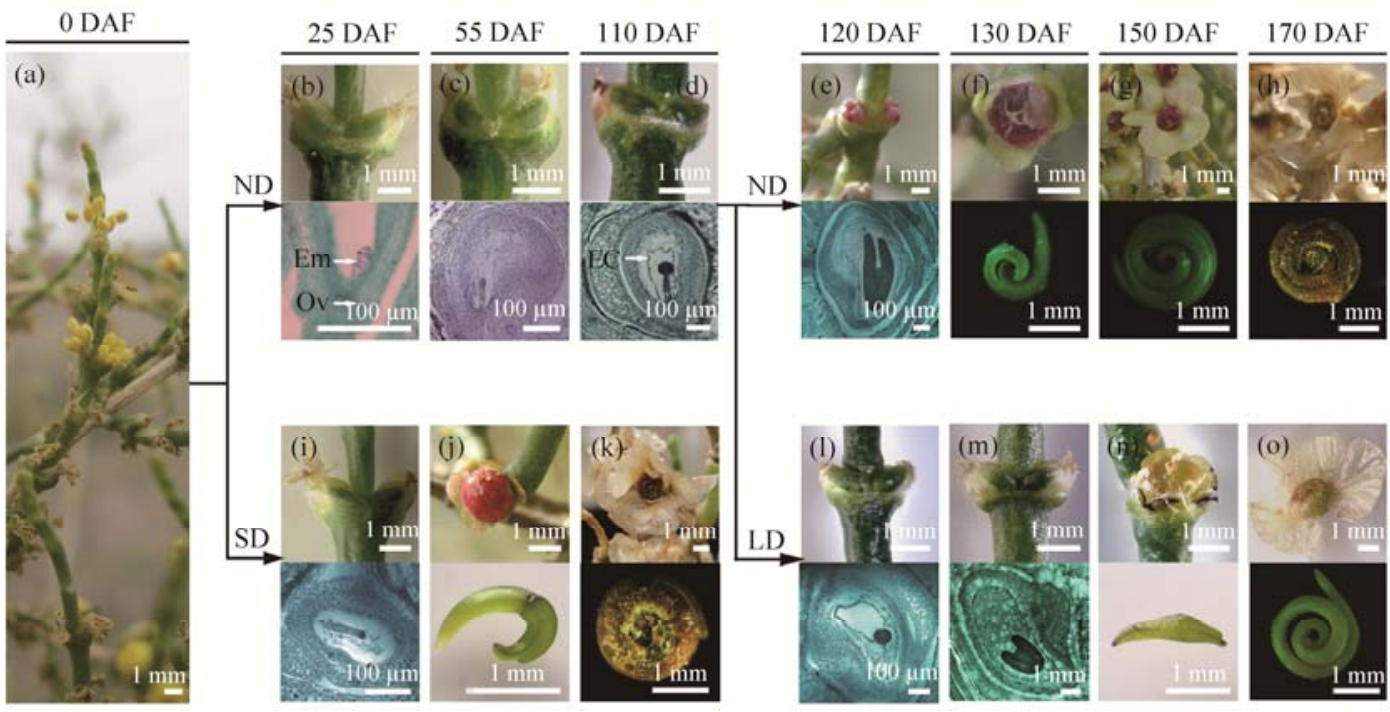

Fig. 6 Morphological and anatomic characteristics of developing fruits of $H$. ammodendron under different daylength (ND, SD, and LD) treatments. Ov, ovule; Em, embryo; Ec, endosperm cell. (a) blooming period; (b, c, i) the early stage of ovary development; $(\mathrm{d}, \mathrm{l}, \mathrm{m})$ the early stage of ovary enlargement; (e) ovary enlargement stage; (f, j, n) the early stage of fruit wing development; (g, o) the fruit wing development stage; (h, k) the fruit maturation stage. 
demonstrated that short day accelerated the fruit development progress of $H$. ammodendron after flowering.

From 14 August (day length, 14.0 h, 110 DAF) onward, LD treatment was conducted on the plants growing under natural conditions. No obvious morphological changes in fruits between the 120 and 130 DAF under LD treatment were observed, whereas the ovary under ND treatment (control) had augmented visibly on 120 DAF. When fruit wings under LD treatment (150 DAF) began to be visible, the fruit wings under ND treatment had been approximately $2-3 \mathrm{~mm}$ long and presented a regular five-flap. When the fruit wings under LD treatment (170 DAF) presented a regular five-flap, the fruits under ND treatment had matured. The results indicated that long day treatment even after fruits had developed for a long time could delay their development progress.

\section{Discussion}

Dormancy in plants is defined as a temporal growth cessation of a meristem. All the classifications of dormancy are related to the organ and/or tissue where the inhibition of growth takes place (Lang, 1987). Zygote dormancy is a common phenomenon in angiosperms and is usually short. But, zygote dormancy can be more prolonged when the endosperm is nuclear (Johri and Srivastava, 2001; Hu, 2005). In this study, the anatomic results indicated that the fruit of $H$. ammodendron was not dormant after flowering. Instead, the fruit developed rather slowly (Fig. 2). Therefore, it is more appropriate to define the delay of fruit development as a slow post-flowering reproductive development (SPRD) phenomenon. Our results showed that it was the day length that caused the SPRD phenomenon of $H$. ammodendron and that $14.0 \mathrm{~h}$ was the most likely day length threshold that can accelerate the reproductive development of $H$. ammodendron. The similar phenomena are also found in annual Amaranthaceous plants of Ceratocarpus arenarius, Salsola brachiata and Atriplex aucheri (Quan, 2012), but rather rare in other species. For example, Diptychocarpus strictus and Tamarix ramosissima evolved different life-history and developed reproductive strategies to adapt to harsh summer ( $\mathrm{Lu}$ et al., 2010; Yan et al., 2011). Our results also suggested that $H$. ammodendron may have developed a SPRD to adapt to the harsh environment in deserts.

The phenomenon of day length variations affecting the development process and the behaviors of organisms is called as photoperiodism (Garner and Allard, 1920; Hayama and Coupland, 2004). It has been proved that the photoperiodism controls many aspects of plant development, such as plant height and reproductive organs (Morandi et al., 1988; Han et al., 2006; Jiang et al., 2011). The phenomenon that the ovary of $H$. ammodendron does not swell after flowering in spring until at the end of August was proposed to be caused by high air temperature and/or drought in soil in summer (Zhang, 2002). However, this study proved that the photoperiodism was the reason behind the SPRD phenomenon of $H$. ammodendron. To simply put, longer day was able to delay the reproductive development progress after flowering, while shorter day was able to accelerate the reproductive development progress (Fig. 6).

The day length threshold is $14.0 \mathrm{~h}$ that can accelerate the reproductive development of $H$. ammodendron. Interestingly, under natural growth conditions, the day length threshold that caused $H$. ammodendron to gradually start SPRD phenomenon at mid-May was the same as that gradually released it from SPRD phenomenon at mid-August. The photo-thermal interaction may explain this interesting phenomenon. In natural conditions, temperature is always associated with photoperiod. Soybean is a typical plant used for the study of photoperiod effects (Han, 2007). Photoperiod is the determining factor of floral transition, and temperature determines soybean development progress. Shorter day length combined with higher temperature is the most effective way to accelerate soybean development. On the contrary, the combination of longer day length and higher temperature can delay the development (Han, 2007). In the present study, under natural conditions, longer day length (>14.0 h critical time) combined with higher temperature from mid-May to mid-August delayed the development of $H$. ammodendron for about 3 months until day length was less than $14.0 \mathrm{~h}$. At the same time, the combination of shorter day length and higher temperature accelerated the fruit development to complete seed maturity. 
The onset of flowering in plants is regulated by multiple environmental and endogenous factors, mainly including photoperiod, vernalization, temperature, age, autonomous pathway, and gibberellin (Fornara et al., 2011; Wang, 2014; Zhang et al., 2016). Autonomous pathway played an important role in ensuring the plant to flower eventually (Glover, 2007). In comparison with those relatively well-known environmental factors that control the photoperiod and vernalization pathways, it is poorly known how the activity of the autonomous pathway is regulated (Quesada et al., 2003). The flowering period of $H$. ammodendron has not changed significantly from 1974 to 2014 (Shi et al., 2014; Han et al., 2015; Jiang et al., 2017), implying that the autonomous pathway of its flowering was rarely affected by environmental conditions.

Additionally, the reproductive strategy, SPRD, is also found to be adopted by H. ammodendron in the other desert regions in northwestern China, such as in the Taklimakan Desert and the Tengger Desert (Zhang, 2002; Sheng et al., 2003). Based on the above results, we proposed a model of photoperiod regulating post-flowering reproductive development progress of $H$. ammodendron as follows: under natural growth conditions, the flowering period of $H$. ammodendron occurs from late April to mid-May. After blooming, its ovary presents a SPRD phenomenon until late August or early September. Afterwards, with cool weather and appropriate precipitation, the ovary of $H$. ammodendron begins to enlarge, and its fruit begins to grow rapidly and matures in October (Figs. 2 and 3). However, under the artificial conditions, the plants of $H$. ammodendron after flowering were treated with a short day length $(<14.0 \mathrm{~h} / \mathrm{d})$, their ovaries would be accelerated in development progress and developed rapidly (Figs. 6i-k), showing no SPRD phenomenon. Moreover, the plants of H. ammodendron that had developed to the early stage of ovary enlargement on $110 \mathrm{DAF}$ were placed under a long day length $(>14.0 \mathrm{~h})$ condition, their fruit development would be delayed (Figs. 6l-o).

\section{Conclusions}

The fruit of $H$. ammodendron was not dormant after flowering. Instead, they developed very slowly. We defined the slow or delayed fruit development as a SPRD phenomenon. Photoperiod was the pivotal environmental factor regulating the SPRD phenomenon. The day length threshold was $14.0 \mathrm{~h}$. The fruit development could be delayed when the day length is longer than $14.0 \mathrm{~h}$ and be accelerated when the day length is shorter than $14.0 \mathrm{~h}$. The SPRD phenomenon is the strategy of $H$. ammodendron to survive in the harsh desert habitats. Our findings will enrich our knowledge in understanding the diverse reproductive strategies adopted by desert plants in response to adverse circumstances and will also provide an insight into the ways to accelerate the seed production of elite $H$. ammodendron genotypes.

\section{Acknowledgements}

This study was financially supported by the National Natural Science Foundation of China (31260181) and the National Science and Technology Ministry of China (2012GB2G400497). We thank Professor WANG Qingya from College of Life Science, Nanjing Agricultural University for the assistance in anatomy experiments.

\section{References}

Brock M A. 1983. Reproductive allocation in annual and perennial species of the submerged aquatic halophyte Ruppia. The Journal of Ecology, 71(3): 811-818.

Cheng L R, Wang Y, Wang C B, et al. 2011. Genetic analysis and QTL detection of reproductive period and post-flowering photoperiod responses in soybean. Theoretical and Applied Genetics, 123(3): 421-429.

Fornara F, de Montaigu A, Coupland G. 2011. SnapShot: Control of flowering in Arabidopsis. Cell (http://www.science direct.com/science/article/pii/S0092867410004411) 550-550. e2.

Garner W W, Allard H A. 1920. Effect of the relative length of day and night and other factors of the environment on growth and reproduction in plants. Journal of Agricultural Research, 18: 553-606.

Glover B J. 2007. The autonomous pathways for floral inhibition and induction. In: Glover B J. Understanding Flowers and 
Flowering. Oxford: Oxford University Press, 35-43.

Guo Q S, Cong Z F, Wang C L. 2009. Ecological Studies of Haloxylon and Cistanche deserticola. Beijing: Science Press, 14. (in Chinese)

Han F G, Xu X Y, Yu Q S, et al. 2015. Responds of reproductive phenology of typical sand-fix plants to climate change in the oasis-desert transitional zone in Minqin, Gansu, China. Journal of Desert Research, 35(2): 330-337. (in Chinese)

Han T F, Wu C X, Tong Z, et al. 2006. Postflowering photoperiod regulates vegetative growth and reproductive development of soybean. Environmental and Experimental Botany, 55(1-2): 120-129.

Han T F. 2007. Photoperiodism in soybean. In: Wang J L, Guo Q Y. Contemporary Soybean Research in China. Beijing: Jindun Press, 211-220. (in Chinese)

Hayama R, Coupland G. 2004. The molecular basis of diversity in the photoperiodic flowering responses of Arabidopsis and rice. Plant Physiology, 135(2): 677-684.

Hu S Y. 2005. Reproductive Biology of Angiosperms. Beijing: Higher Education Press, 204-226. (in Chinese)

Jiang J F, Liang C H, Yang H, et al. 2017. Influence of temperature and precipitation on phenology of desert plant Haloxylon ammodendron and Cornulaca alaschanica in recent ten years. Journal of Arid Land Resources and Environment, 31(2): 141-146. (in Chinese)

Jiang Y, Wu C X, Zhang L X, et al. 2011. Long-day effects on the terminal inflorescence development of a photoperiod-sensitive soybean [Glycine max (L.) Merr.] variety. Plant Science, 180(3): 504-510.

Johri B M, Srivastava P S. 2001. Reproductive Biology of Plants. Berlin: Springer, 256.

Lang G A. 1987. Dormancy: A new universal terminology. Hortscience, 122(5): 817-820.

Li H P. 2009. Plant Microtechnique ( $2^{\text {nd }}$ ed.). Beijing: Science Press, 9-47 (in Chinese)

Li J, Zhao C Y, Song Y J, et al. 2010. Spatial patterns of desert annuals in relation to shrub effects on soil moisture. Journal of Vegetation Science, 21(2): 221-232.

Lu J J, Tan D Y, Baskin J M, et al. 2010. Fruit and seed heteromorphism in the cold desert annual ephemeral Diptychocarpus strictus (Brassicaceae) and possible adaptive significance. Annals of Botany, 105(6): 999-1014.

Ma H, Wang Y F, Cao R. 2004. Study on embryology of Haloxylon ammodendron I. Preliminary observation on microsporogenesis and formation of male gametophytes and apomixis in megasporogenesis. Journal of Desert Research, 24(6): 768-772. (in Chinese)

Morandi E N, Casano L M, Reggiardo L M. 1988. Post-flowering photoperiodic effect on reproductive efficiency and seed growth in soybean. Field Crop Research, 18(4): 227-241.

Pyankov V I, Black C C Jr, Artyusheva E G, et al. 1999. Features of photosynthesis in Haloxylon species of Chenopodiaceae that are dominant plants in Central Asian deserts. Plant and Cell Physiology, 40(2): 125-134.

Quan D J. 2012. The life-history strategies of several Chenopodiaceae annuals. MSc Thesis. Urumqi: Xinjiang Agricultural University, 18-27. (in Chinese)

Quesada V, Macknight R, Dean C, et al. 2003. Autoregulation of FCA pre-mRNA processing controls Arabidopsis flowering time. The EMBO Journal, 22(12): 3142-3152.

Shamsutdinov Z S, Ubaidullaev S R. 1988. Distribution of poa bulbosa l. and carex pachystylis gay in the limits of phytogenous field of haloxylon aphyllum. Problems of Desert Development, 1: 38-43.

Sheng J H, Liu H Y, Pan D Z, et al. 2003. Study on phonological phases of Haloxylon ammodendron (C. A. Mey.) Bunge. Review of China Agricultural Science and Technology, 5(3): 60-63. (in Chinese)

Sheng Y, Zheng W H, Pei K Q, et al. 2005. Genetic variation within and among populations of a dominant desert tree Haloxylon ammodendron (Amaranthaceae) in China. Annals of Botany, 96(2): 245-252.

Shi W, Zhao Y F, Pan B R, et al. 2014. Phenological behaviour of desert plants in response to temperature change: A case study from Turpan eremophytes Botanical Garden, northwest China. Pakistan Journal of Botany, 46(5): 1601-1609.

Su P X, Cheng G D, Yan Q D, et al. 2007. Photosynthetic regulation of $\mathrm{C}_{4}$ desert plant Haloxylon ammodendron under drought stress. Plant Growth Regulation, 51(2): 139-147.

Tobe K, Li X M, Omasa K. 2000. Effects of sodium chloride on seed germination and growth of two Chinese desert shrubs, Haloxylon ammodendron and H. persicum (Chenopodiaceae). Australian Journal of Botany, 48(4): 455-460.

Tursunov Z, Matyunina T E, Kiseleva G K, et al. 1989. Seed reproduction of the main forest-forming species of the central Asian deserts. Problems of Desert Development, 2: 53-57.

Wang J W. 2014. Regulation of flowering time by the miR156-mediated age pathway. Journal of Experimental Botany, 65(17): 4723-4730.

Wang X M, Yang D Y, Tian Y Z, et al. 2009. Genetic relationship between parasitized and non-parasitized Haloxylon ammodendron in the Alxa Desert. Journal of Systematics and Evolution, 47(3): 255-262. 
Wei Y, Wang X Y. 2006. Role of winged perianth in germination of Haloxylon (Chenopodiaceae) seeds. Acta Ecologica Sinica, 26(12): 4014-4018. (in Chinese)

Xu H, Li Y, Xu G Q, et al. 2007. Ecophysiological response and morphological adjustment of two Central Asian desert shrubs towards variation in summer precipitation. Plant, Cell \& Environment, 30(4): 399-409.

Yan C, Wei Y, Yang M L. 2011. Comparative germination of Tamarix ramosissima spring and summer seeds. EXCLI Journal, 10: 198-204.

Yang W B, Feng W, Jia Z Q, et al. 2014. Soil water threshold for the growth of Haloxylon ammodendron in the Ulan Buh desert in arid northwest China. South African Journal of Botany, 92: 53-58.

Yu T. 2012. Study on H. ammodendron for response to land surface temperature stress, pivotal control factor of reproductive dormancy after flowering, technology of artificial regeneration plantations using simple protective device to resist stress. PhD Dissertation. Nanjing: Nanjing Agricultural University, 111-112. (in Chinese)

Yu T, Ren C, Zhang J P, et al. 2012. Effect of high desert surface layer temperature stress on Haloxylon ammodendron (C. A. Mey.) Bunge. Flora, 207(8): 572-580.

Zhang L Y. 2002. Haloxylon ammodendron Bunge and H. Persicum Bunge ex Boiss in Xinjiang desert. Plants, (4): 4-6. (in Chinese)

Zhang M, Zhu J X, Wang L, et al. 2016. Progress of stress-induced flowering in plants. Chinese Journal of Biotechnology, 32(10): 1301-1308. (in Chinese)

Zhang Y M, Chen J, Wang L, et al. 2007. The spatial distribution patterns of biological soil crusts in the Gurbantunggut Desert, Northern Xinjiang, China. Journal of Arid Environments, 68(4): 599-610.

Zhang Z X. 2008. Dendrology ( $2^{\text {nd }}$ ed.). Beijing: China Forestry Publishing House, 53-55. (in Chinese) 